\title{
Analyzing Dislocations with Virtual Dark Field Images Reconstructed from Electron Diffraction Patterns
}

\author{
E.F. Rauch and M. Véron \\ SIMAP Laboratory, CNRS-Grenoble INP, BP 46101 rue de la Physique, 38402 Saint Martin d'Hères, \\ France
}

Dark field images are routinely produced in transmission electron microscopy by selecting a specific diffracted beam with the objective aperture while the incident beam illuminates a large area of the thin foil. Similar pictures are produced in STEM mode by scanning the condensed beam over the region of interest and reconstructing the microstructure thanks to the signal collected with an annular detector. For crystallized materials such images are particularly useful to highlight structural features like second phase particles, nanograins or dislocations.

In both cases, the diffracted information is filtered thanks to a given technical component that provides a limited range of possible settings. Typically, few apertures, which differ by their diameter, are available in common Transmission Electron Microscopes (TEM). In scanning mode, the camera length is the only practical parameter that may be adapted for sorting the signals. In some cases it could be of interest to extend these capabilities to non-standard situations. For example, small precipitates that promote faint diffracting beams could be highlighted by collecting the intensities of not only one but several of them. Also, some crystallographic texture components could be detected by separating the annular dark field detector in distinct sectors. Such developments are technically feasible but too specific to dedicated situations to lead to reasonable and low cost solutions.

To that respect, acquiring the entire diffracted signal with a spatially resolved detector - e.g.: a CCD camera - and sorting numerically the information out of this complete set of material may reveal refined features and produce uncommon views of the substructure. It is this approach that is used to construct so-called virtual bright field (VBF) or dark field (VDF) images with the orientation mapping system developed by the authors [1] and known as the ASTAR $^{\mathrm{TM}}$ tool from NanoMEGAS [2]. The TEM is set in nano-beam mode and the incident electron beam is scanned over the area of interest at a rate of typically 100 points per second thanks to a dedicated hardware control system of the TEM deflecting coils. The spot patterns are collected with an external camera that point on the TEM phosphorous screen and stored in the computer memory for post processing.

A virtual bright field image is obtained by measuring on every successive diffraction pattern the intensity of the transmitted beam (Fig.1). Because the signal is truncated by the limited depth of the collected images (i.e.: 8 bit $=256$ grey levels), the transmitted beam intensity cannot be entirely retrieved. Therefore, the value is approximated by averaging the levels of all pixels contained in a disk of a user-selected diameter and located at the centre of the diffraction pattern. The procedure is similar to the insertion of a virtual aperture in the beam path (Fig.1.a). Consequently, it mimics the production of a STEM type bright field image. For a dark field image, the virtual aperture is located on the relevant diffracting spot. The diameter of the aperture is adapted to the intensity of the diffracting signal and may be reduced to a single pixel. [2] 
The resulting numerical micrographs bear strong similarities with the classical bright and dark field images obtained on TEMs and may be used to analyse the structural features of the observed materials. Figure 2 reproduces the Burgers vector analysis for dislocations in deformed ferrite. The same area is imaged by centring the virtual dark field aperture on successively two different diffraction vectors $\mathbf{g}$. The best contrast is obtained with the strongest diffracting beam that is $\mathbf{g}=[11-2]$. For $\mathbf{g}=[1-16]$, the quality is certainly less good but sufficient to notice the appearance of additional segments with respect to the previous image. These dislocation lines turns out to be screw segments with a [111] Burgers vectors that gives rise to the usual $\mathbf{g} \cdot \mathbf{b}=0$ extinction condition for one of the VDF images. The beauty of the procedure is that the Burgers vector analysis was performed off-line and afterwards by using stored information while this particular scan was not performed for that particular purpose.

It may be easily figured out that because the aperture is computer-generated, there is no limit in size, number and shape of 'holes' in the aperture. This flexibility allows additional characterisation routes that will be discussed.

[1] E.F. Rauch and M Veron, J. Mater. Sci. Eng. Tech. 36 (2005) 552-556.

[2] E.F. Rauch, J. Portillo, S. Nicolopoulos, D. Bultreys, S. Rouvimov and P.Moeck, Zeitschrift für Kristallographie: (2010) Vol. 225, issue 2-3 pp. 103-109

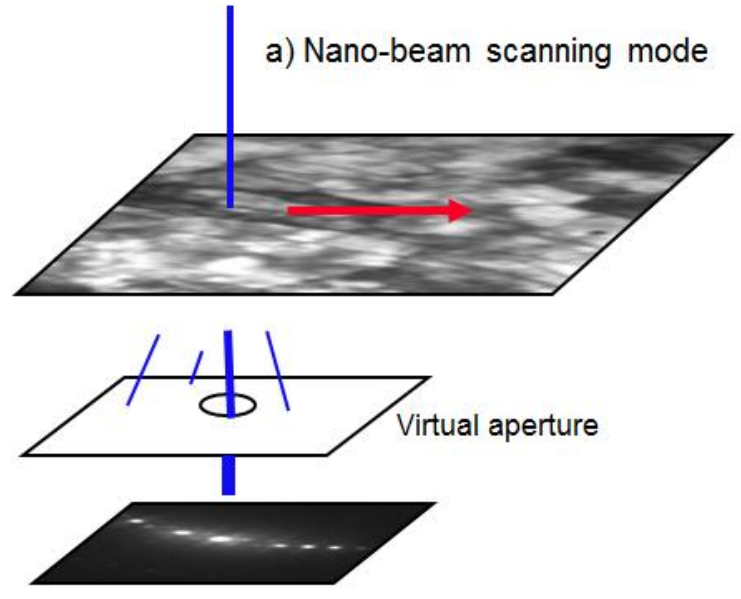

b) acquisition of a stack of diffraction patterns

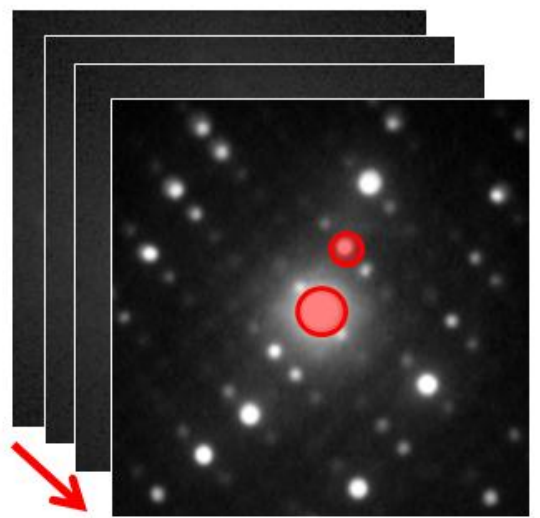

Figure 1. a) Diffraction patterns are collected while the focussed beam is scanned over the sample, b) all patterns are stored in the computer memory. VBF and VDF are obtained by averaging the intensity within disks that are centred on the relevant diffracting spot.
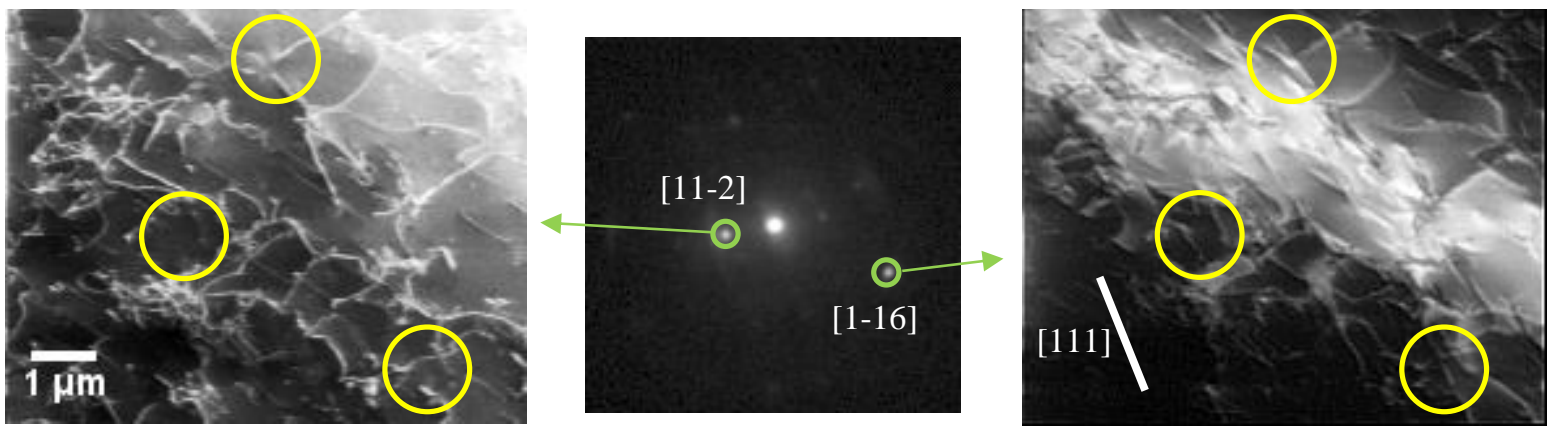

Figure 2. Virtual Dark Field micrographs of deformed low carbon steel. Dislocations with the [111] Burgers vector (surrounded) do not appear if the [11-2] diffracting spot is selected to generate the image. 